\title{
Improving the Safety of Endoscopy in Pregnancy: Approaching Gravidity with Gravitas
}

\author{
Mitchell S. Cappell ${ }^{1,2}$ \\ Published online: 25 August 2020 \\ ○) Springer Science+Business Media, LLC, part of Springer Nature 2020
}

Keywords Pregnancy · Inflammatory bowel disease · Crohn's disease · Ulcerative colitis · Fetal safety · Endoscopy · Flexible sigmoidoscopy $\cdot$ Colonoscopy $\cdot$ Therapeutic endoscopy

Any medical intervention that is used during pregnancy is subject to intensive scrutiny due to its potential for harming not only the mother but the fetus, the latter being extraordinarily susceptible to drugs or procedures that may have little effect on adults. The difficulty of assessing risk is amplified by the observation that some of the ill effects of medical interventions emerge several years after birth, requiring long-term observation. In numerous circumstances, such as profuse GI hemorrhage or severe IBD flares, endoscopy can be lifesaving. For gastroenterologists, the question arises as to whether an endoscopic procedure performed during pregnancy has the potential for harming the mother or the fetus. Although these questions are addressed by performing clinical studies, conducting this research during pregnancy is subject to stringent demands. First, even relatively small increases in the incidence of major congenital deformities after endoscopy could constitute an endoscopic contraindication. Statistically excluding small yet significant increases in the incidence of major fetal complications after endoscopy requires large study populations, since pregnant mothers not undergoing endoscopy have a low baseline risk of fetal injury. Second, studies of endoscopic safety in non-pregnant patients normally require short-term follow-only, but such studies in pregnant patients must follow-up pregnancy outcome to postpartum, up to 9 months after endoscopy, to

Mitchell S. Cappell

mscappell@yahoo.com; Mitchell.Cappell@Beaumont.edu

1 Division of Gastroenterology and Hepatology, William Beaumont Hospital, MOB \#602, 3535 W. Thirteen Mile Rd, Royal Oak, MI 48073, USA

2 Division of Gastroenterology and Hepatology, Oakland University William Beaumont School of Medicine, MOB \#602, 3535 W. Thirteen Mile Rd, Royal Oak, MI 48073, USA assess newborn health, and arguably for years thereafter in order to detect subtle, but significant, neuropsychiatric deficits that may manifest only years after birth. Third, an ideal, controlled randomized trial of endoscopic safety during pregnancy raises ethical concerns and is impractical since endoscopy is rarely performed during pregnancy. Fourth, the pregnant study population is diverse, with patients widely split among endoscopic performance during first, second, or third trimesters; variable endoscopic indications; and a broad range of severity of the medical status of pregnant mothers at endoscopy.

In order to address these issues, Ludvigsson et al. [1] published a landmark study in 2017 demonstrating fetal safety among 3052 patients undergoing gastrointestinal (GI) endoscopy during pregnancy by statistically analyzing a huge national registry in Sweden. This large Swedish study provided the robust statistical power needed to investigate the significance of uncommon, yet important, pregnancy complications. For example, the relative risk of major congenital malformations after endoscopy versus no endoscopy during pregnancy was 0.98 , with a narrow 95\%-confidence-interval (0.82-1.19) [1]. Contrariwise, the largest prior published medical chart study [2] encompassed only 83 patients undergoing esophagogastroduodenoscopy (EGD) during pregnancy. Does publication of this large Swedish study preclude a need for further studies?

Absolutely not! Multiple considerations, arranged hierarchically from minor to major, demonstrate that the study by Ko et al. [3] published in this issue of Digestive Diseases and Sciences is necessary and important. First, the scientific method demands independent studies to confirm findings [4] in order to exclude unintentional researcher bias to "prove" positive results and to exclude methodological errors. Second, since the Ko study was based on an American population, fetal safety of endoscopy could differ from 
a Swedish study due to differing patient demographics, physician training, and differences in healthcare systems between continents. Such differences are, moreover, likely exaggerated when comparing one tertiary American hospital (University of California, San Francisco), versus 100 Swedish hospitals, and when comparing data obtained during mostly different time periods. Third, the American study incorporated 47 patients solely undergoing sigmoidoscopy, whereas the Swedish study combined 1109 sigmoidoscopies and colonoscopies. Sigmoidoscopy may be safer than colonoscopy during pregnancy due to its lesser invasiveness, reduced need for anesthesia or sedation, and simpler endoscopic technique. Fourth, the studies used different methodologies: medical chart review versus a national registry. National registry studies require much less labor compared with chart-based studies, primarily because a national registry has preexistent patient data already abstracted from medical charts.

Yet, registry studies have limitations, since they generally contain fewer patient data than medical chart reviews. Moreover, medical charts can be re-reviewed to mine new data if necessary, whereas registries contain fixed, preexistent databases that cannot be expanded to mine new parameters.

Fortunately, these twofold limitations of registry studies are mostly mitigated by the Swedish study demonstrating that endoscopy is generally not associated with increased rates of poor fetal outcomes and is highly likely to be relatively safe to the fetus in utero. Nevertheless, a positive study demonstrating a significantly increased risk of poor fetal outcomes would require analysis of numerous additional clinical parameters, such as the use of sedatives and anesthetics, endoscopic indications, and endoscopic therapy, in order to identify predictors of poor outcomes.

The Ko study has important strengths (Table 1). First, it focuses on one highly clinically relevant endoscopic indication: assessing IBD severity by sigmoidoscopy. About onethird of patients with previously clinically stable IBD experience flares during pregnancy [3]. Patients with suspected flares in the general population, including non-pregnant females during their childbearing years, routinely undergo sigmoidoscopy or colonoscopy in order to endoscopically and histologically assess IBD severity, extent, and activity to determine need for drugs such as biologics to suppress flares. Second, this work focuses on sigmoidoscopy which, as aforementioned, entails less theoretical fetal risks than colonoscopy. The need for endoscopic assessment is even greater in pregnant mothers with IBD than in the general population due to the hidden, second patient, the fetus in utero, who experiences particularly poor outcomes if the mother has active disease.

Yet, endoscopic assessment is constrained by concern for fetal safety as tragically illustrated by the experience with thalidomide [5], administered to suppress maternal nausea and vomiting during the first trimester of pregnancy, which was responsible for an epidemic of about 4000 cases of phocomelia, a severe, congenital malformation of deformed or rudimentary limbs [6]. The US FDA had approved thalidomide for all patients, including pregnant patients, based on safety in clinical trials in nonpregnant patients, without testing fetal safety in pregnant females. After this medical catastrophe, the United States FDA Drug Regulation Act of 1962 codified that safety of drugs, and later of endoscopy, during pregnancy must be directly tested by clinical studies in pregnant patients [6]. The Hippocratic Injunction of "First, do no harm!" was extended to "First, prove no fetal harm!" by mandating studies on pregnant subjects prior to prescribing drugs or performing endoscopy.

Second, medicolegal concerns limit clinical data on fetal safety of endoscopy during pregnancy. A pervasive fear is that poor fetal outcomes, such as major congenital fetal anomalies occurring after endoscopy during pregnancy could generate malpractice suits that might yield astronomical jury awards due to the lifetime disability incurred by an infant. Endoscopists and anesthesiologists may limit performance of endoscopy during pregnancy in America due to medicolegal concerns and lacking or inadequate clinical guidelines. This policy perpetuates a vicious cycle of avoiding even potentially lifesaving endoscopy due to potential fetal risks leading to few, small, and unconvincing clinical studies and the avoidance of endoscopies during pregnancy, even when strongly indicated. The remedy is more, larger, and better clinical studies to provide endoscopists evidence-based guidelines to promote a virtuous cycle encouraging more endoscopies during pregnancy in order to yield more clinical data and analysis.

In conclusion, clinical studies provide the basis for promulgating evidence-based guidelines regarding endoscopy during pregnancy. The Ko study should prompt a new systematic review, and promulgation of new endoscopic guidelines stratified according to endoscopic test, endoscopic indication, and patient status; graded as strongly, moderately, weakly, or not indicated; and rated according to statistical power of the clinical studies. 
Table 1 Study strengths and limitations

Major strengths

1. Reports severity, activity, location, and type of IBD found at sigmoidoscopy to determine need for biologic therapy

2. Semiquantitatively characterizes IBD severity by Mayo endoscopy score

3. Reports trimester of pregnancy during which sigmoidoscopy performed

4. Analyzes only pregnant patients with known or suspected IBD

5. Single hospital chart review rather than national database registry

6. Study conducted at major academic medical center

7. Adds to the growing literature addressing fetal safety of sigmoidoscopy during pregnancy

Minor strengths

1. Data independently abstracted by 2 investigators, and discrepancies resolved by third investigator

2. Reports lifestyle factors of history of alcoholism and recreational drug use. Reports smoking habits and use of folic acid use during pregnancy

3. Reports data about bowel preparation

4. Reports pregnancy outcome: premature delivery, low birth weight, type of delivery, oligo- or poly-hydramnios, chorioamnionitis, and premature rupture of membranes. Also reports gestational diabetes and gestational hypertension

5. Reports data on sedation for sigmoidoscopy

6. Analyzes noninvasive markers of inflammation, including leukocyte count, hemoglobin level, CRP, ESR, and fecal calprotectin. Correlates these markers with endoscopic findings of severity of inflammation

Study limitations

1. Retrospective study

2. No control group

3. Moderately sized patient population $(\mathrm{N}=48$ patients, including patients undergoing colonoscopy)

Minor study issues

1. Does not report patient benefits accrued from initiating biologic therapy—remitting IBD flare—as determined by: remission of bloody diarrhea, decrease in other IBD symptoms, increased hemoglobin, lowered leukocyte count, decreased ESR, decreased CRP, decreased fecal leukocytes, decreased fecal calprotectin level, and disappearance of fecal occult blood

2. Aside from IBD severity and pregnancy-related disorders (e.g., gestational diabetes), does not report baseline major illnesses (such as renal insufficiency) or overall maternal medical health (e.g., American Society of Anesthesiologists [ASA] score, or APACHE [Acute Physiology and Chronic Health Evaluation] II) score

3. Does not report maternal/fetal drug toxicity from initiating biologic therapy after sigmoidoscopy

4. Does not report outpatient versus inpatient status at time of performing sigmoidoscopy

5. Does not report the location of the sigmoidoscopy: ambulatory surgery center, hospital endoscopy suite, operating room, or intensive care unit

6. Does not report neonatal health beyond delivery. Subtle neurodevelopmental delays may only become apparent years after birth

7. Does not report whether any sigmoidoscopies were therapeutic (e.g., dilatation of Crohn's stricture or colonic polypectomy)

8. Does not report neonatal deaths $<28$ days after delivery

9. Does not quantify the severity of hematochezia or melena in patients with these indications for sigmoidoscopy by reporting mean number of units of packed erythrocytes transfused or acute hemoglobin decline. This limitation is substantially mitigated by reporting mean baseline hemoglobin levels

10. Does not report data on fetal monitoring during sigmoidoscopy (generally not performed during sigmoidoscopy)

11. Does not report data on endoscopic complications even if transient (e.g., intra-procedural hypoxia, hypotension, or cardiac arrhythmias)

12. Does not report whether endoscopy had to be aborted or shortened because of complications (e.g., conversion of intended colonoscopy to sigmoidoscopy)

13. Does not report data addressing suspected maternal procedure-related complications based on events occurring during or $\leq 24 \mathrm{~h}$ after procedure

$I B D$ inflammatory bowel disease, $C R P$ C-reactive protein, $E S R$ erythrocyte sedimentation rate 
Funding None.

\section{Compliance with Ethical Standards}

Conflict of interest None. Dr. Cappell did not receive any financial support for writing this editorial. In particular, Dr. Cappell, as a consultant of the United States Food and Drug Administration (FDA) Advisory Committee for Gastrointestinal Drugs, affirms that this paper does not discuss any proprietary, confidential, pharmaceutical data submitted to the FDA. Dr. Cappell was also a member of the speaker's bureau for AstraZeneca and Daiichi Sankyo, co-marketers of Movantik until more than 2 years ago. Dr. Cappell received a one-time honorarium from Shire and Mallinckrodt more than 2 years ago. This work does not discuss any drug manufactured or marketed by AstraZeneca, Daiichi Sankyo, Shire, or Mallinckrodt.

\section{References}

1. Ludvigsson JF, Lebwohl B, Ekbom A, Kiran RP, Green PH, Höijer J, Stephansson O. Outcomes of pregnancies for women undergoing endoscopy while they were pregnant: a nationwide cohort study. Gastroenterology. 2017;152:554-563.e9. doi: https://doi. org/10.1053/j.gastro.2016.10.016. Epub 2016 Oct 20. PMID: 27773807.

2. Cappell MS, Colon VJ, Sidhom OA. A study of eight medical centers of the safety and clinical efficacy of esophagogastroduodenoscopy in 83 pregnant females with follow-up of fetal outcome with comparison control groups. Am J Gastroenterol. 1996;91:348-354.

3. Ko MS, Rudrapatna V, Avila P, Mahadevan U. Safety of flexible sigmoidoscopy in pregnant patients with known or suspected inflammatory bowel disease. Dig Dis Sci. (Epub ahead of print). doi: https://doi.org/10.1007/s10620-020-06122-8.

4. Gauch HG Jr. Scientific Method in Practice. Cambridge University Press. ISBN 978-0-521-01708-4 435 p.

5. Curran WI. The thalidomide tragedy in Germany: the end of a historic medicolegal trail. N Engl J Med. 1971;284:481-482.

6. Yaffe SJ. Introduction. In: Briggs GG, Freeman RK, Towers U, Forinash AB, eds. Drugs in Pregnancy and Lactation: A Reference Guide to Fetal and Neonatal Risk. 11th ed. Philadelphia: Walters Kluwer; 2017:13-19.

Publisher's Note Springer Nature remains neutral with regard to jurisdictional claims in published maps and institutional affiliations. 